\title{
Spatial-Temporal Characteristics Analysis of Urban Air Quality and Its Evolution Pattern in Henan Province
}

\author{
Kaiguang Zhang*, Mingting Ba, Hongling Meng and Yanmin Sun \\ Zhengzhou Normal University, Zhengzhou 450044, China \\ ${ }^{*}$ Corresponding author
}

\begin{abstract}
Urban air pollution is one of the most urgent problems to be solved in the process of China's economic development and urbanization. Aiming at the spatial-temporal evolution problem of air quality in Henan province, this paper, based on the daily average data of urban air quality index of Henan province from 2014 to 2017, utilizes geostatistical analysis and spatial analysis methods to study the spatial and temporal distribution characteristics, as well as its evolution mechanism. The results show that: The urban air quality of Henan province is mainly Grade II and Grade III, there are obvious variations among cities, the air qualities of southern cities are significantly better than that of northern cities; From 2014 to 2017, the urban air quality has been gradually improved, the serious pollution process has been gradually reduced, the process duration has been gradually shortened, the air quality improvement in the southern cities is significantly better than that in the central and northern cities, but Luoyang in the western part of the province has gradually increased; the urban air quality shows seasonal distribution characteristics, autumn is the best season, from winter to autumn, air quality gradually improves. The urban air quality also presents the monthly distribution characteristics of low in the middle and high at two ends with a local extreme point in June, and August is the best month for air quality. The urban air quality index shows agglomeration characteristics, and the high value regions gradually shrink to the north, forming two heavily polluted regions centered on Zhengzhou and Anyang respectively.
\end{abstract}

Keywords-Urban air quality; spatial distribution; spatialtemporal evolution; spatial aggregation; Henan Province

\section{INTRODUCTION}

Since reform and opening, the rapid development of China's economy has been promoted the rapid accumulation of material wealth and the rapid increase of urbanization level, meanwhile, the inefficient use of resources and population agglomeration have also been causing serious ecological problems in many urbanized regions, especially atmospheric environmental problems. Air pollution in Chinese cities has seriously affected the atmospheric environment and public health of urban regions, and has hindered regional sustainable development. [1-5].

In recent years, the study of air quality based on geoscience vision has mainly focused on the variation characteristics analysis of air quality at different scales and in typical regions, and the influencing factors analysis of air quality, has achieved many valuable results. In fact, a city's air quality has its own evolution characteristics, impacted by its natural environment, there are some certain correlations among cities. Understanding the spatial and temporal evolution of urban air quality is not only conducive to the scientific understanding of the characteristics of urban air pollution changes in the region, but also could provide some scientific reference for the formulation and implementation of regional prevention and control measures[3-10].

Air quality Index (AQI) is a quantitative data describing the short-term air quality situation and changing trend of a region. Based on the impact of various pollutants on human health, ecology and environment, according to the Environmental Air Quality Standard, AQI simplifies the monitored main air pollutant concentrations into a single conceptual index[0,500], classifies air quality as six grades, the larger the index, the more serious the pollution situation, the greater harm to the human body [2,3,5].

Based on the daily average data of urban AQI in Henan province from 2014 to 2017, this paper utilizes geostatistical analysis and spatial analysis methods to study the spatial and temporal distribution characteristics, as well as its evolution mechanism in order to provide some scientific reference for the regional air pollution prevention.

\section{DATA AND RESEARCH METHODS}

\section{A. Research Area Overview and Data.}

Henan province $\left(31^{\circ} 23^{\prime} \mathrm{N}-36^{\circ} 22^{\prime} \mathrm{N}, 110^{\circ} 21^{\prime} \mathrm{E}-116^{\circ} 39^{\prime} \mathrm{E}\right)$ is located in the central part of China, consists of 17 cities with a total area of 167 thousand $\mathrm{km} 2$, its three sides as the north, west and south are semi-circular surrounded by Taihang, Funiu and Tongbai mountains, its central and east regions are Huanghuaihai alluvial plain[11,12]. the data used in the study mainly includes the city spatial distribution data coming from National Earth System Science Data Sharing Infrastructure (http://www.geodata.cn/data/publisher.html), the daily average air quality index of 17 cities (from 2017.1.1-2017.12.30) derived from the key city air quality daily report data of the Ministry of Environmental Protection of the People's Republic of China (http://datacenter.mep.gov.cn), the air quality historical data of 17 cities released by the China's air quality 
online monitoring and analysis platform released (https://www.aqistudy.cn/historydata/).

\section{B. Digital Characteristics of Air Quality Index}

The digital characteristic mean $E$ of AQI distribution presents average air quality level in the study region, the standard deviation $\sigma$ and the range $R$ describe the difference degree of the samples, the larger $\sigma$ and $R$ means the more significant variation of the regional air quality[12].

$$
\begin{gathered}
E=\frac{1}{n} \sum_{i=1}^{n} A_{i}, \sigma^{2}=\frac{1}{n-1} \sum_{i=1}^{n}\left(A_{i}-E\right)^{2}, \\
R=\max \left(A_{i}\right)-\min \left(A_{i}\right),
\end{gathered}
$$

\section{Centrality Analysis of Air Quality Index}

Although the amount of atmospheric pollutant emissions is the main factor determining urban air quality, the regional topographic conditions, wind direction, wind speed, precipitation and temperature have some certain impacts on the spread of the pollutants. Regional transmission and transport is one of the important features of air pollution, the air quality of a region is affected by the pollution conditions of its adjacent regions, and there are some certain correlations among districts. The AQI center $B$ of a region defines as the AQI weighted average of city geographic coordinates,

$$
B=\left(\frac{A_{i}}{\sum A_{i}} x_{i}, \frac{A_{i}}{\sum A_{i}} y_{i}\right),
$$

where $A_{i}$ and $\left(x_{i}, y_{i}\right)$ are the geographic coordinates of city and the AQI of city $i$.

\section{SPATIAL-TEMPORAL EVOLUTION ANALYSIS OF URBAN AIR QUALITY INDEX IN HENAN}

\section{A. The Spatial Distribution Analysis of Urban Air Quality in Henan Province}

In the 1461 days of observation, Henan air quality is mainly lightly and moderately polluted, about 817 days accounting for 55.92\%. From 2014 to 2017, the air quality continues to improve, the average urban AQI decreases from 117.56 to 97.62 , the good \& moderate days increases from 34 to 100 , the heavily $\&$ severely polluted days decreases from 127 to 60. The province's air pollution shows regional characteristics, and the AQI is quite different among cities.

In 2014, Henan AQI and the average urban AQI respectively are 180.76 and 117.56 , respectively, and corresponding standard deviation and range respectively are (75.93, 456.01) and (47.51, 322.94). The good \& moderate days is 34 , the heavily \& severely polluted days is 27 . There are 13 serious pollution processes, in which the longest lasting is of 7 days.

In order to facilitate comparison, this paper divides the regional AQI index 4 grades, as high value region $(\geq 122)$, subhigh value region $([106,122))$, median region $([110,106))$, and low value region $(\leq 106)$.

The annual average urban AQI shows regional characteristics (Figure 1a), the northernmost city Anyang is the high city with 133, Zhengzhou(122.68) and the cities south of it, as Xucheng(122.69), Pingdingshan(122.18), Nanyang (122.67), Luohe (119.20) and Zhumadian (120.00), forms the high value region.

There are some differences in the good \& moderate days(Figure 1b), the heavily \& severely polluted days(Figure 1c) , and the urban annual average AQI (Figure 1a) among cities. The minimum of the good \& moderate days appearing in Anyang is about 136 days, and the maximum appearing in Sanmenxia is about 222 days, the range reaches 86 days. The distribution of the good \& moderate days is coupled with the urban average AQI in Anyang, Zhengzhou, Xuchang, Luohe and Nanyang, there are certain difference in Pingdingshan (180 days) and Zhumadian (184 days), The maximum of the heavily \& severely polluted days appearing in Anyang is of 54 days, the minimum is its adjacent city, Hebi, is of 18 days, Anyang, Pingdingshan (43), Xuchang (43) and Zhumadian (46) are coupled with the urban average AQI, but There are certain differences in Zhengzhou (39) and Nanyang ( 37), the most significant difference city is Luohe (only 31 ).

In general, Anyang and Xuchang are the most polluted cities in the year, with the highest the annual average AQI, the least the good \& moderate days, and the most the heavily \& severely polluted days.

In 2015, Henan AQI and the average urban AQI respectively are 175.23 and 114.02, respectively, and corresponding standard deviation and range respectively are $(78.40,433.00)$ and $(48.74,329.47)$. The good \& moderate days is 49 , the heavily \& severely polluted days is 24 . There are 11 serious pollution processes, in which the longest lasting is of 6 days.

The high value region shows a zonal distribution from Anyang to Pingdingshan along northeast-southwest direction, includes Xingxiang and Zhengzhou, in where the distribution of the good \& moderate days and the heavily \& severely polluted days are coupled with that of the average urban AQI(Figure 1d). In the sub-high value region, Xuchang and Luohe have better annual average AQI and fewer good \& moderate days, Jiaozuo has better annual average AQI and more good \& moderate days(Figure 1e). The maximum of the good \& moderate days appearing in Hebi is of 233 days, the minimum appearing in Zhengzhou is of 134 days(Figure 1f), where has the maximum of the heavily \& severely polluted days about 46 days, the minimum of the heavily \& severely polluted days appearing in Nanyang is of 14 days.

In general, except for Hebi, Zhengzhou and the cities 


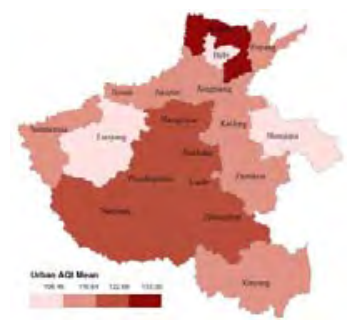

(a) 2014 Urban AQI Mean

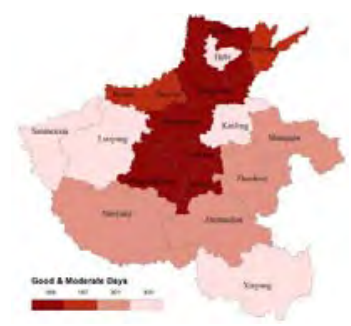

(e) 2015 G\&M Days

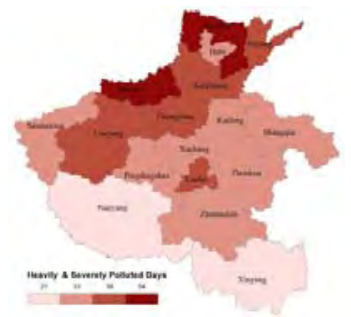

(i) 2016 H\&S Polluted Days

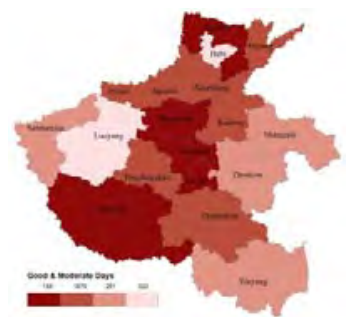

(b) 2014 G\&M Days

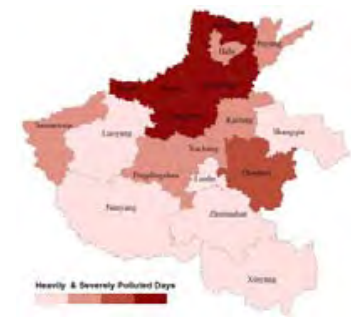

(f) 2015 H\&S Polluted Days

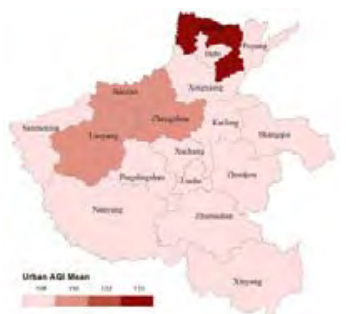

(j) 2017 Urban AQI Mean

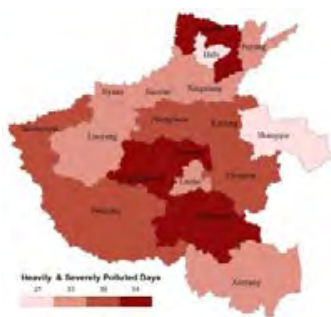

(c) 2014 H\&S Polluted Days

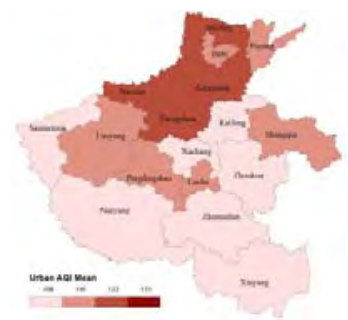

(g) 2016 Urban AQI Mean

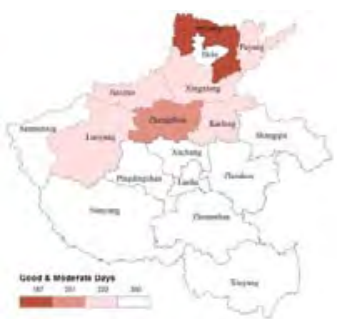

(k) 2017 G\&M Days

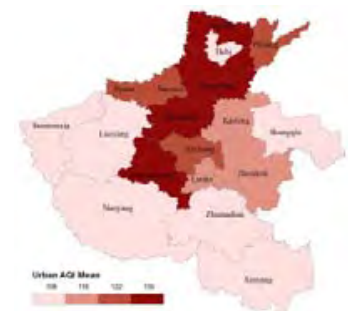

(d) 2015 Urban AQI Mean

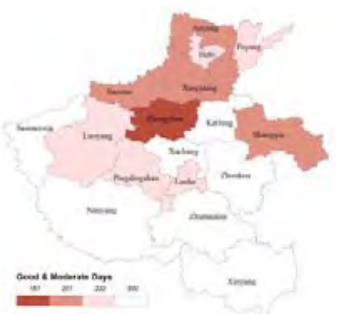

(h) 2016 G\&M Days Mean

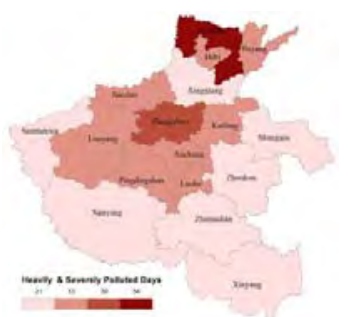

(l) 2017 H\&S Polluted Days

FIGURE I. THE SPATIAL DISTRIBUTIONS OF URBAN AQI IN HENAN PROVINCE FROM 2014 TO 2017

north of it are the most polluted cities in the year, where has the highest of the average annual AQI, the least of the good \& moderate days, and the most of the heavily \& severely polluted days.

In 2016, Henan AQI and the average urban AQI respectively are 156.32 and 108.21 , respectively, and corresponding standard deviation and range respectively are (82.21, 450.00,) and (55.90, 392.07). The good \& moderate days is 98 , the heavily \& severely polluted days is 24 . There are 11 serious pollution processes, in which the longest lasting is of 4 days.

According to the classification standard of the previous two years, the high value region basically disappears, the highest value region of this year is equivalent to the previous sub-high value region. This region of this year includes Anyang(121.97), Puyang(120.06), Xingxiang(128.22), Jiaozuo(118.68) and Zhengzhou(118.19), where the average urban AQI gradually increases from north to south(Figure 1g), the distribution of the good \& moderate days and the heavily \& severely polluted days are coupled with that of the annual average urban AQI(Figure 1h, 1i), the good \& moderate days is inversely proportional to the average urban AQI, and the heavily \& severely polluted days (Figure $1 \mathrm{i}$ ) is proportional to the average urban AQI. The maximum of the good \& moderate days appearing in Xinyang is of 265 days, the minimum appearing in Zhengzhou is of 189 days, the range reaches 76 days. The maximum of the heavily \& severely polluted days appearing in Anyang is of 46 days, the minimum of the heavily \& severely polluted days appearing in Xinyang is of 14 days.

Overall, in 2015 and 2016, except for Hebi, Zhengzhou and the cities north of it are the most polluted cities in the whole year, where has the highest of the average annual AQI, the least of the good \& moderate days, and the most of the heavily \& severely polluted days.

In 2017, Henan AQI and the average urban AQI respectively are 145.64 and 102.91, respectively, and corresponding standard deviation and range respectively are (72.96, 412.11) and $(47.33,285.12)$. The good \& moderate days is 100 , the heavily \& severely polluted days is 22 . There are 11 serious pollution processes, in which the longest lasting is of 4 days.

The high value region reappears in Anyang with the average urban AQI 122.05, the sub-high region does not present. Zhengzhou(111.71), Luoyang(1151.55) and Jiaozuo (111.01) form a median value region, the remaining cities all have entered low value region(Figure $1 \mathrm{j}$ ). The distribution of the good \& moderate days is coupled with that of the annual average urban AQI(Figure 1k). In the median value region, the good \& moderate days is inversely proportional to the average urban AQI.

Xinyang, the southernmost city, enjoys the maximum of the good \& moderate days(287) and the minimum of the 


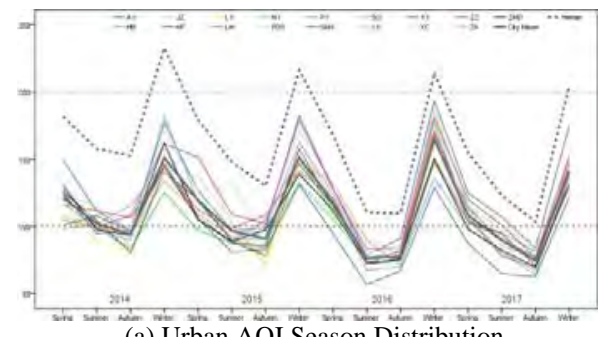

(a) Urban AQI Season Distribution

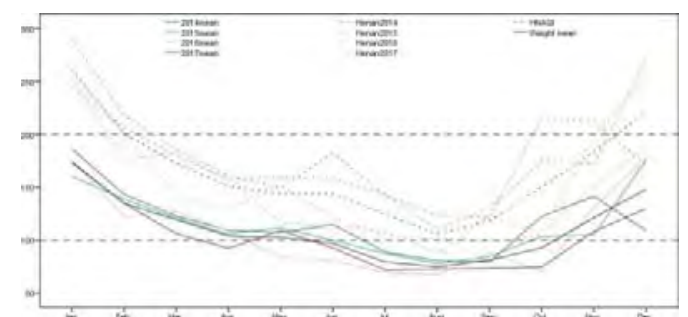

(b) Urban AQI Month Distribution

FIGURE II. THE TEMPORAL DISTRIBUTION OF URBAN AQI IN HENAN PROVINCE FROM 2014 TO 2017

heavily \& severely polluted days(10); Anyang has the maximum of the heavily \& severely polluted days (44) and the minimum of the good \& moderate days(180).

In general, Anyang is the most polluted cities in the whole year, where has the highest of the average annual AQI, the least of the good \& moderate days, and the most of the heavily \& severely polluted days.

\section{B. The Temporal Distribution Analysis of Urban Air Quality in Henan Province}

From 2014 to 2017, the province annual average AQI and the urban annual average AQIs gradually decrease, except for Luoyang with the increasing annual average AQI. The air quality improvement has significant differences, the declining degree of the southern cities, as Xinyang, Nanyang, Zhumadian, Xuchang and Luohe are all more than 20.

The province AQI and urban AQIs show obvious seasonal characteristics (Figure 2a), which gradually decrease from spring to autumn, and reach the annual maximum value in winter. The decrease rate of the second time period is significantly slower than that of the first time period, and the increase rate of the third time period is significantly greater than the first two time periods. From 2014 to 2017, the air qualities of province and cities are gradually improving, the improvement degree in autumn is significantly higher than that in other seasons, as autumn-summer -spring-winter.

In spring, Zhoukou AQI increases gradually, and other cities decrease. Compared with 2014, the biggest reduction city is Zhumadian (31.34), followed by Xuchang (27.06), Sanmenxia (22.00) and Anyang (24.37), Luoyang is basically the same (0.24). In summer, compared with 2014, Luoyang and Zhengzhou AQI increase by 14.17 and 1.01 respectively, and other cities have decreased, especially Xinyang (43.21) and Luohe(33.84). In autumn, compared with 2014, Jiaozuo and Luoyang AQI increase by 4.76 and 0.42 respectively, and other cities have decrease, especially Zhoukou (48.30), followed by Luohe (36.65), Xinyang (33.32), Nanyang (29.91), Xuchang (28.10), Zhumadian (23.52) and Shangqiu (20.67). In winter, compared with 2014, Hebi, Puyang, Luohe, Jiaozuo, Luoyang and Kaifeng AQI increase by 11.9, 3.11, 2.44, 1.28, 1.25 and 0.33 respectively, and other cities decrease, especially Nanyang(45.3), followed by Pingdingshan(33.69), Zhumadian(31.27)and Xinyang(24.21).

The province AQI and the urban AQI presents the monthly distribution characteristics of low in the middle and high at two ends with a local extreme point in the middle(Figure 2b).
The AQI maximums appear in 1 and 12 months, 2 times each; the extreme points appears 3 times in June, 1 times in May, shows 6665 distribution; the AQI minimums appear in August and September 2 times each, shows 9988 distribution. Compared with 2014, the two indices increase in May and December, and decrease in other months. From the monthly average, the AQIs are the highestin January, gradually decreases from January to August, reach the minimum in August, and then gradually increase, the AQIs in December are about the average of January and February.

\section{The Centrality Analysis of Urban Air Quality in Henan Province}

The geometric center of the 17 cities is located at (11346'52.98"E, 34²4'13.03"N), with 5 cities in each of 1-3 quadrant and 2 cities in the 4th quadrant (Figure 3a).
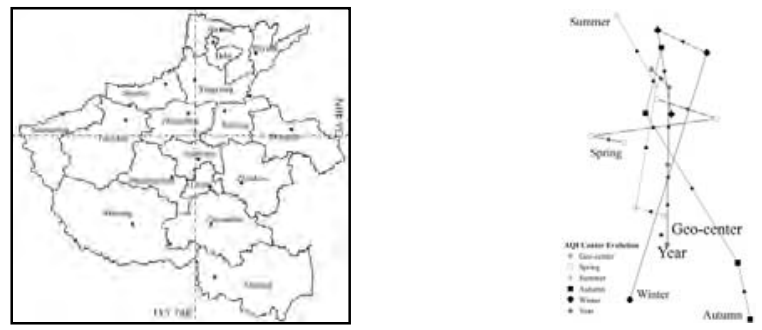

FIGURE III. THE CENTER DISTRIBUTIONS OF URBAN AQI IN HENAN PROVINCE FROM 2014 TO 2017

In 2014, the AQI center is located in the south of the geometric center. From 2014 to 2017, the AQI center gradually moves to the northwest. In the first two periods, the moving is along the north direction, and the moving speed is basically the same (about $3.92 \mathrm{KM}$ ), which is about 3 times of the 3rd period. In the 3rd period, the moving azimuth angle is about $315^{\circ}$, points to the geometric center of Zhengzhou, Luoyang and Jiaozuo (Figure 3b).

In the view of the seasonal evolution, the AQI center of 2014 spring is located $5 \mathrm{~km}$ northwest of the geometric center. From 2014 to 2017, the center gradually moves $2.18 \mathrm{~km}$ to the north, meanwhile moves east-west around the geometric center, with an amplitude of $6.30 \mathrm{~km}$. The AQI center of 2014 summer is located at $0.98 \mathrm{~km}$ north of the geometric center. From 2014 to 2017, the center gradually moves to the northwest by $10.31 \mathrm{~km}$, and the north-south movement is about four times that of the east-west amplitude. The AQI center of 2014 autumn is located $6.14 \mathrm{~km}$ southeast of the geometric center. From 2014 to 2017, the center gradually moves to the 
northwest by $14.32 \mathrm{~km}$, and the north-south movement is about 2.84 times that of the east-west movement. The AQI center of 2014 winter is located at $3.61 \mathrm{~km}$ southwest of the geometric center. From 2014 to 2015, the center moves to the northeast by $12.95 \mathrm{~km}$, which is the maximum migration during the study period. From 2015 to 2016, the center moves to northwest by $2.71 \mathrm{~km}$, from 2016 to 2017, the AQI center moves to southeast by $4.21 \mathrm{~km}$.

\section{CONCLUSION}

This paper, based on the daily average data of urban AQI of Henan province from 2014 to 2017, utilizes geostatistical analysis and spatial analysis methods to study the spatial and temporal distribution characteristics, as well as its evolution mechanism. The results show that:

- The urban air quality of Henan province is mainly Grade II and Grade III, there are obvious variations among cities, the air qualities of southern cities are significantly better than that of northern cities; From 2014 to 2017, the urban air quality has been gradually improved, the serious pollution process has been gradually reduced, the process duration has been gradually shortened, the air quality improvements in the southern cities are significantly better than that in the central and northern cities, but Luoyang in the western part of the province has gradually increased;

- The urban air quality shows seasonal distribution characteristics, and autumn is the best season, from winter to autumn, air quality gradually improves. The urban air quality also presents the monthly distribution characteristics of low in the middle and high at two ends with a local extreme point in June, and August is the best month for air quality.

- The urban air quality index shows agglomeration characteristics, and the high value regions gradually shrink to the north, forming two heavily polluted regions centered on Zhengzhou and Anyang respectively.

\section{REFERENCE}

[1] Jiang Lei, Zhou Haifeng, Bai Ling, et al. The analysis of socio-economic factors of air quality index (AQI) based on the perspective of the exponential decay effects. Acta Scientiae Circumstantiae, 2018, 38(1):390-398

[2] Zhang Nan Nan, Wu Sun Ze, Li Yi Fan. Evaluation of the ambient air quality improvement in China based on percentile method. Acta Scientiae Circumstantiae, 2018,38(5):2082-2089.

[3] Lin Xueqin, , WANG Dai. Spatio-temporal variations and socioeconomic driving forces of air quality in Chinese cities. Acta Geographica Sinica, 2016, 71(8): 1357-1371.

[4] Wang Zhenbo, Fang Chuanglin, Xu Guang, et al. Spatial- temporal characteristics of the PM 2.5 in China in 2014. Acta Geographica Sinica, 2015,70(11): 1720-1734.

[5] Xue An, Geng Enze. Region Division Study of PM2. 5 Pollution in Cities of China Based on Complex Networks. Journal of Basic Science And Engineering, 2018,23(Supplement):68-78.

[6] Zhang Yinjun, Chen Xi, Xie Gaodi, et al. Pollution status and spatial distribution of PM 2.5in China. Resources Science, 2015,37(7):13391346.
[7] Peng Jian, Chen Sha, Lü Huiling, et al. Spatiotemporal patterns of remotely sensed PM 2.5 concentration in China from 1999 to 2011. Remote Sensing of Environment, 2016, 174: 109-121.

[8] Ning Miao Sun Yamei Lli Wei. Study on the Method of City Air Quality Attainment Judgment. Environment and Sustainable Development, 2017,42(1):154-157.

[9] Zhang Shengling, Wang Yao, Li Yue, et al. Does regional haze opinion affect air quality?. Journal of Arid Land Resources and Environment,2018,32(8):100-106.

[10] Qiao X, Jaffe D, Tang Y, et al. Evaluation of air quality in Chengdu, Sichuan Basin, China: Are China's air quality standards sufficient yet? Environ. Monit. Assess., 2015, 187(5): 1-11.

[11] Zhang Kaiguang, Ba Mingting, Sun Yanmin, et al. Study on spatial distribution characteristics of intra-city agriculture sub-sector valuesadded growth rates in Henan province. Advances in Engineering Research,2015,33:167-171.

[12] Chen Yanguang. Mathematical Methods for Geography. Beijing: Science Press,2017.

[13] Zhang Kaiguang, Ba Mingting, Meng Hongling. Spatial-temporal characteristics of Henan Highway traffics network accessibility and its evolution pattern. Science of Surveying and Mapping. 2017,42(6):87-92. 